\title{
Cost Analysis of Cot-Side Screening Methods for Neonatal Hypoglycaemia
}

\author{
Matthew J. Glasgow ${ }^{a}$ Jane E. Harding a Richard Edlin ${ }^{b}$ \\ for the CHYLD Study Team

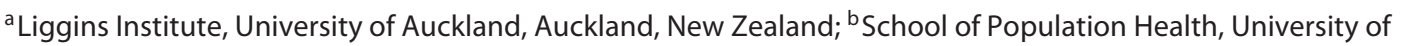 \\ Auckland, Auckland, New Zealand
}

\section{Keywords}

Neonatal hypoglycaemia $\cdot$ Screening $\cdot$ Glucometer $\cdot$ Health economics $\cdot$ Cost analysis

\begin{abstract}
Background: Babies at risk of neonatal hypoglycaemia are often screened using cot-side glucometers, but non-enzymatic glucometers are inaccurate, potentially resulting in over-treatment and under-treatment, and low values require laboratory confirmation. More accurate enzymatic glucometers are available but at apparently higher costs. Objective: Our objective was to compare the cost of screening for neonatal hypoglycaemia using point-of-care enzymatic and non-enzymatic glucometers. Methods: We used a decision tree to model costs, including consumables and staff time. Sensitivity analyses assessed the impact of staff time, staff costs, probability that low results are confirmed via laboratory testing, false-positive and false-negative rates of nonenzymatic glucometers, and the blood glucose concentration threshold. Results: In the primary analysis, screening using an enzymatic glucometer cost NZD 86.94 (USD 63.47) while using a non-enzymatic glucometer cost NZD 97.08 (USD 70.87) per baby. Sensitivity analyses showed that using
\end{abstract}

an enzymatic glucometer is cost saving with wide variations in staff time and costs, irrespective of the false-positive level of non-enzymatic glucometers, and where $\geq 78 \%$ of low values are laboratory confirmed. Where non-enzymatic glucometers may be less costly (e.g., false-negative rate exceeds $15 \%)$, instances of hypoglycaemia will be missed. Reducing the blood glucose concentration threshold to $1.94 \mathrm{mmol} / \mathrm{L}$ reduced the incidence of hypoglycaemia from 52 to $13 \%$, and the cost of screening using a non-enzymatic glucometer to NZD 47.71 (USD 34.83). Conclusions: In view of their lower cost in most circumstances and greater accuracy, enzymatic glucometers should be routinely utilised for point-ofcare screening for neonatal hypoglycaemia.

(c) 2018 S. Karger AG, Basel

\section{Introduction}

Neonatal hypoglycaemia is a common metabolic condition which, even if asymptomatic, carries a risk of poor neurological outcome [1], although the severity and duration of hypoglycaemia that may result in poor neurological outcomes remain unclear [2]. The incidence is approximately $5-15 \%$, but can be up to $50 \%$ in those with

\section{KARGER}

(c) 2018 S. Karger AG, Basel

E-Mail karger@karger.com

www.karger.com/neo
Jane E. Harding

Liggins Institute, University of Auckland

Private Bag 92019

Grafton, Auckland 1142 (New Zealand)

E-Mail j.harding@ auckland.ac.nz 
risk factors [3, 4]. Most cases (75\%) of hypoglycaemia occur within the first $24 \mathrm{~h}$ after birth [3], with screening and management usually confined to the first week.

Initial management of neonatal hypoglycaemia usually involves feeding or buccal dextrose gel. If the infant does not tolerating feeding, or there is an inadequate response, admission to the neonatal intensive care unit (NICU) for intravenous dextrose is usually indicated [5]. There are significant direct costs associated with treating hypoglycaemia, particularly if the infant is admitted to NICU. Although the extent to which treatment of asymptomatic infants changes the outcome is uncertain [2,4], if diagnosis and treatment of severe cases is inadequate, neurological complications, including developmental delay [6], cognitive impairment [7, 8], sensory impairment [7], seizures [7, 9], and cerebral palsy $[1,10]$, may require ongoing management and support, resulting in considerable long-term financial and societal cost.

Most neonatal hypoglycaemia is asymptomatic, and although the risk of serious complications in asymptomatic infants is uncertain, the screening of infants at risk is recommended, requiring regular measurement of blood glucose concentrations [11]. Recommended thresholds for diagnosis and treatment of neonatal hypoglycaemia vary considerably $[4,5,11,12]$, but $2.6 \mathrm{mmol} / \mathrm{L}$ (47 mg/ $\mathrm{dL})$ is widely used as a treatment target [13-15].

Laboratory enzymatic (e.g., glucose oxidase) methods of glucose measurement are considered the gold standard. Cot-side methods of glucose measurement provide convenience and rapid results, but often utilise non-enzymatic glucometers employing reflectance colorimeter and electrode methods, e.g., HemoCue ${ }^{\circledR}$ Glucose 201 system (HemoCue, Ängelholm, Sweden), StatStrip ${ }^{\circledR}$ Glucose Xpress $^{\mathrm{TM}}$ device (Nova Biomedical, Waltham, MA, USA), and ACCU-CHEK ${ }^{\circledR}$ Inform II device (Roche Diagnostics, Basel, Switzerland). Most non-enzymatic glucometers were designed for use in adults, with precision optimised for the normoglycaemic and hyperglycaemic ranges [16]. In the hypoglycaemic range, they have been shown to both overestimate and underestimate blood glucose concentrations [16]. Nevertheless, they are widely used to screen for and diagnose neonatal hypoglycaemia $[14,17]$ because they are commonplace for glucose monitoring in adults and older children with diabetes, are easy to use, and require only small amounts of blood [18]. Existing recommendations state that the use of non-enzymatic devices in neonates should be minimised, and results should be confirmed by laboratory testing if used as a screening method $[5,16]$.
Point-of-care glucometers are available that use an enzymatic reaction (glucose oxidase or glucose dehydrogenase) to generate a current proportional to glucose concentration, including the Abbott $^{\circledR}$ i-STAT ${ }^{\circledR}$ HANDHELD portable blood analyser (Abbott. Princeton, NJ, USA) and AlereTM epoc ${ }^{\circledR}$ Reader and Host2 Mobile Computer blood analysis system (Alere, Waltham MA, USA). Enzymatic glucometers have greater accuracy [5] and therefore do not require confirmation by laboratory testing, but the higher initial and apparent operating costs have limited their uptake. Nevertheless, it is possible that use of enzymatic glucometers might reduce the overall cost of neonatal glucose screening by avoiding the need for laboratory re-analysis. Improved accuracy may also reduce under-treatment and over-treatment.

We therefore undertook a cost analysis using a decision tree comparing bedside testing using non-enzymatic glucometers, which require abnormal results to be verified by laboratory testing, to enzymatic glucometers, which can directly inform management without re-testing.

\section{Methods}

A decision tree modelling approach was selected because the health events in question (detected hypoglycaemia) are either present or absent at each point of measurement, and occur relatively closely together [19]. The evaluation was undertaken from the perspective of a postnatal ward/hospital.

Incidence data used in the primary analysis to model the proportions of at-risk infants who develop neonatal hypoglycaemia, and those who experienced recurrent episodes, was extracted from the raw data from the Sugar Babies Study [3]. This study recruited infants at risk of hypoglycaemia: late preterm (35-37 weeks' gestation), small ( $<10$ th percentile or $<2,500 \mathrm{~g}$ ), large ( $>90$ th percentile or $>4,500 \mathrm{~g}$ ), infant of diabetic mother, or other. Blood glucose concentrations were measured $1 \mathrm{~h}$ after birth, then every 3-4 h before feeds for the first $24 \mathrm{~h}$, and every $6-8 \mathrm{~h}$ for the subsequent $24 \mathrm{~h}$ [20]. Hypoglycaemia was defined as a blood glucose concentration of $<2.6 \mathrm{mmol} / \mathrm{L}$.

The decision tree model covered approximately a 1-week time horizon and compared two different approaches to point-of-care screening for neonatal hypoglycaemia in newborn infants identified as being at risk:

1. A non-enzymatic glucometer is used for the initial blood sample analysis. If a result is below the diagnostic threshold for hypoglycaemia, a repeat blood sample is processed using a laboratory analyser. Management is guided by the laboratory result.

2. A point-of-care enzymatic reaction glucometer is used for all blood glucose measurements and management is guided by the results. No confirmatory laboratory analysis is performed.

Initial screening of infants at risk of neonatal hypoglycaemia is performed using either an enzymatic or a non-enzymatic point-ofcare blood glucose analyser (Fig. 1). If results are below the thresh- 


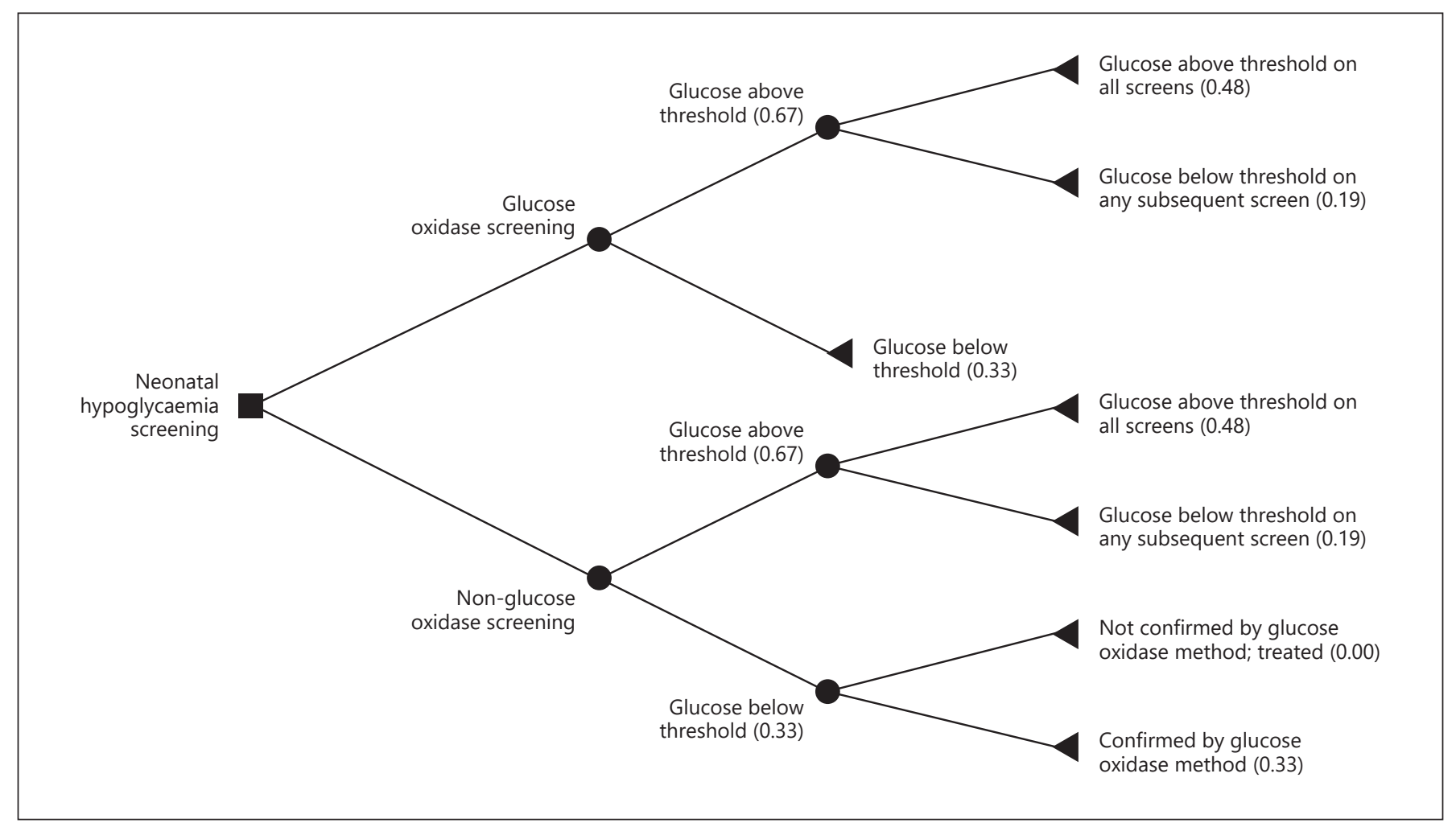

Fig. 1. Decision tree for cot-side hypoglycaemia screening (with probabilities).

old using an enzymatic point-of care device, the infant is treated for hypoglycaemia. If results are below the threshold using nonenzymatic point-of-care tests, they may be confirmed by laboratory testing (as per protocol) or treatment may proceed without confirmation (deviation from protocol).

In the primary analysis, the decision tree algorithm incorporated a glucose concentration threshold of $2.6 \mathrm{mmol} / \mathrm{L}$, a cut-off value that is widely used internationally [5] and consistent with New Zealand practice guidelines [21]. Data were censored when an infant was admitted to NICU, because beyond that time glucometers are rarely used, and the costs of blood glucose measurements are very small compared to overall costs.

The costs associated with the two screening approaches fall into two main categories: consumables and staff time. Consumables include reagents involved in the analysis, which in handheld devices are incorporated into a strip or cartridge, the lancet used to draw blood, and capillary tubes used to collect the blood sample for analysis by enzymatic-reaction-based glucometers. Staff time includes time for sample collection, which is slightly longer with enzymatic glucometers because blood is collected in a capillary tube before being applied to the cartridge or card, whereas with non-enzymatic glucometers blood is directly applied to a test strip. We used estimates of staff time required to perform blood glucose measurement based on reports from multiple ward and laboratory staff who regularly perform these tests. In a clinical laboratory samples are processed in batches, and costs were derived by divid- ing the number of glucose tests performed on the laboratory analysers by the number of samples.

Costs were collected in 2015/2016 New Zealand dollars (USD $1=$ NZD 1.37) (Table 1). Staff costs were based on an hourly rate equivalent to the top of the salary range for a senior registered nurse or midwife (step 5) [22].

To simplify the calculations we assumed that the same type of lancet was used to obtain blood for all testing methods. We assumed that nurses or midwives were the staff taking and analysing all samples, presuming past training in the use of the device. We also assumed that samples collected in micro-tubes were sent to the laboratory using a pneumatic tube or similar, thus excluding any additional costs for staff time required to transport samples to the laboratory.

Sensitivity analyses were undertaken by varying the following parameters, selected because they were key drivers of the model, or because of known variability in practice: staff time to perform a blood glucose test; the probability that guidelines would be followed and that a low blood glucose concentration detected by nonenzymatic testing would be confirmed by laboratory testing; the threshold used to define hypoglycaemia and therefore the requirement for laboratory re-testing; and the accuracy (false-positive and false-negative rates) of non-enzymatic glucometers. 
Table 1. Costs used in the primary analysis

\begin{tabular}{|c|c|c|c|c|c|}
\hline Testing method & Cost of consumables & $\begin{array}{l}\text { Total } \\
\text { consumables/ } \\
\text { processing costs }\end{array}$ & $\begin{array}{l}\text { Collection } \\
\text { resources } \\
\text { (staff time) }\end{array}$ & $\begin{array}{l}\text { Staff } \\
\text { costs }\end{array}$ & $\begin{array}{l}\text { Total cost } \\
\text { per test }\end{array}$ \\
\hline $\begin{array}{l}\text { Abbott i-STAT Handheld } \\
\text { portable blood analyser }\end{array}$ & $\begin{array}{l}\text { Lancet } 0.20 \\
\text { CLINITUBES } 100-\mu \mathrm{L} \text { capillary tubes } \\
143.00 / 250 \\
\text { i-STAT glucose cartridges } \\
143.70 / 25\end{array}$ & 6.70 & $6 \mathrm{~min}$ & 3.30 & 10.00 \\
\hline $\begin{array}{l}\text { Alere }{ }^{\mathrm{TM}} \text { epoc Reader and } \\
\text { Host2 Mobile Computer } \\
\text { blood analysis system }\end{array}$ & $\begin{array}{l}\text { Lancet } 0.20 \\
\text { epoc Care Fill Tubes } 98.00 / 50 \\
\text { epoc BGEM test cards } 376.00 / 50\end{array}$ & 9.68 & $6 \mathrm{~min}$ & 3.30 & 12.98 \\
\hline $\begin{array}{l}\text { Average cost of enzymatic } \\
\text { glucometer }\end{array}$ & & & & & $\begin{array}{l}11.49 \\
\text { (USD 8.39) }\end{array}$ \\
\hline ACCU-CHEK ${ }^{\circ}$ Inform II & $\begin{array}{l}\text { Lancet } 0.20 \\
\text { Test cartridge/strip 21.65/50 }\end{array}$ & 0.64 & $5 \mathrm{~min}$ & 2.75 & 3.39 \\
\hline StatStrip Glucose Xpress ${ }^{\mathrm{TM}}$ & $\begin{array}{l}\text { Lancet } 0.20 \\
\text { Test strip } 60.00 / 100\end{array}$ & 0.80 & $5 \mathrm{~min}$ & 2.75 & 3.55 \\
\hline HemoCue Glucose 201 system & $\begin{array}{l}\text { Lancet } 0.20 \\
\text { Test cartridge } 2.86\end{array}$ & 3.06 & $5 \mathrm{~min}$ & 2.75 & 5.81 \\
\hline $\begin{array}{l}\text { Average cost of non-enzymatic } \\
\text { glucometer }\end{array}$ & & & & & $\begin{array}{l}4.25 \\
\text { (USD 3.10) }\end{array}$ \\
\hline Laboratory analysis & & 1.29 & & 20.00 & $\begin{array}{l}21.29 \\
\text { (USD15.54) }\end{array}$ \\
\hline
\end{tabular}

All costs are in New Zealand dollars (NZD) and converted at a rate of USD $1=$ NZD 1.37. Staff costs are assessed at NZD 33.00/h (USD 24.09/h). Data were acquired by personal communications with New Zealand-based product distributors, public hospital laboratory staff, and research institute staff and records. Pricing information is generally not published publicly due to variances based on scale of purchases and/or existing wider contract relationships. Where cost information was received from more than one source for the same item or service, there was a generally good level of agreement between the values reported. However, caution is required if these findings are generalised to other locations.

\section{Results}

In the scenario used for the primary analysis, approximately half (52\%) of at-risk infants developed hypoglycaemia with one third (33\%) doing so at their first screening test, and the remainder (19\%) on the next test after a single normal test. The mean number of blood glucose tests was 6.0 in at-risk infants who did not have hypoglycaemia, 7.0 in infants with an initial measurement below the threshold, and 11.1 in infants whose first measurement was above the threshold but who had a subsequent measurement below the threshold.

If non-enzymatic glucometer testing is assumed to be accurate and all glucose measurements below the threshold by non-enzymatic testing are confirmed by laboratory testing, then screening using an enzymatic glucometer costs NZD 86.94 (USD 63.47) while screening using a non-enzymatic glucometer costs NZD 97.08 (USD 70.87) per infant screened.
The following sensitivity analyses were performed (Table 2).

\section{Staff Time}

The use of an enzymatic glucometer remains less costly even if the time to test with a non-enzymatic glucometer is reduced ( $2 \mathrm{~min})$, if the time to test with an enzymatic glucometer is increased $(8 \mathrm{~min})$, or if both are simultaneously adjusted to thresholds of around 2 and 7 min, respectively. The non-enzymatic glucometer generally costs less than the enzymatic glucometer when the latter takes staff members 5 min longer to use.

\section{Staff Costs}

The use of enzymatic glucometers remains less costly over a wide range of staff costs up to NZD 56/h, with higher savings increasing for lower hourly staff costs. 
Table 2. Base case analysis and sensitivity analyses

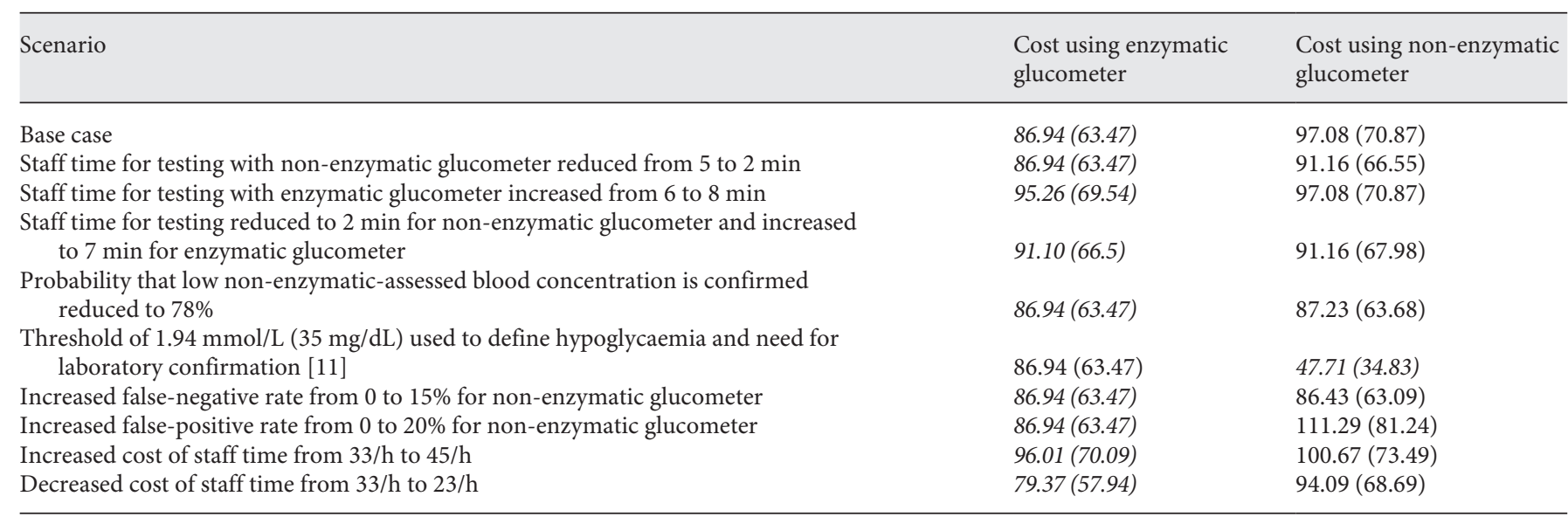

All costs are in New Zealand dollars (NZD) with US dollars (USD) in parentheses. Costs are converted at a rate of USD $1=$ NZD 1.37 . The lowest cost per row is shown in italics.

\section{Confirmation of Results}

Enzymatic glucometers remain less costly if the falsepositive rate of non-enzymatic glucometers is increased beyond zero, with the difference in costs increasing as the false-positive rate increases, as this increases the number of tests requiring laboratory confirmation. At a false-positive rate of $20 \%$, the difference in cost per infant screened is NZD 24.35 (USD 17.77) in favour of enzymatic testing. Enzymatic glucometers are also less costly if the proportion of low blood glucose concentrations detected by a non-enzymatic glucometer followed up with a confirmatory test is reduced to $78 \%$. If fewer than $78 \%$ of low results are re-tested, then non-enzymatic glucometers are less costly, but unconfirmed results may be false positives, each of which would increase over-treatment and associated costs.

\section{Incidence of Hypoglycaemia}

When the blood glucose concentration threshold for definition of hypoglycaemia is reduced to $1.94 \mathrm{mmol} / \mathrm{L}$ [11], the incidence of hypoglycaemia falls to $13 \%$ in our dataset, and the use of a non-enzymatic glucometer is less costly as fewer laboratory confirmatory tests are used.

\section{Accuracy of Non-Enzymatic Glucometers}

Similarly, non-enzymatic glucometers cost more if their false-negative rate is $\leq 15 \%$, but less with a higher false-negative rate (i.e., the incidence of hypoglycaemia is artificially reduced by more than $15 \%$ ), although this is associated with more missed diagnoses.

Cost Analysis of Neonatal Hypoglycaemia Screening

\section{Discussion and Conclusions}

We performed a cost analysis comparing the use of non-enzymatic and enzymatic point-of-care glucometers in screening for neonatal hypoglycaemia. Use of a more accurate enzymatic glucometer results in cost savings compared to using a non-enzymatic glucometer under usual conditions.

Non-enzymatic glucometers are often used to screen for neonatal hypoglycaemia because of their availability and familiarity. The initial purchase price and cost of ongoing use of enzymatic glucometers are commonly cited as reasons for persisting with screening using non-enzymatic glucometers. However, that does not take into account either the immediate implications of using a screening method with a level of accuracy that requires re-testing to verify abnormal results, or the long-term repercussions of failing to identify and treat cases of hypoglycaemia. The requirement for additional blood sampling to confirm low results also increases the number of invasive procedures performed on the newborn infant and may delay treatment where confirmation is required by laboratory testing. In preterm babies, numbers of painful procedures are themselves associated with neurodevelopmental impairment [23]. In addition, false-positive results can result in over-treatment, thus increasing costs as well as separating the infant from its mother, and interfering with the establishment of breastfeeding [24], which may in turn have adverse long-term health and cognitive effects $[25,26]$. 
Sensitivity analyses showed that the use of an enzymatic glucometer remained less expensive than a nonenzymatic glucometer, even if testing with a non-enzymatic glucometer takes less than half the time ( 3 vs. 7 $\mathrm{min})$. In practice, once staff members are trained and familiar with cot-side devices, most report little difference in the time required for testing.

Our primary analysis takes the widely recommended position that results below the threshold measured by a non-enzymatic glucometer will be confirmed by laboratory analysis of a repeat sample $[5,11]$. In practice this is not always done [14]. Sensitivity analysis showed that enzymatic glucometers would be cost saving provided at least $78 \%$ of non-enzymatic low results are confirmed. If more than $22 \%$ of low results are not confirmed, screening with a non-enzymatic glucometer has lower shortterm costs, but resulting missed hypoglycaemia and overtreatment have associated burdens for the infant, family, and healthcare system.

Recommendations for the diagnostic blood glucose concentration threshold for hypoglycaemia vary between guidelines. If a lower blood glucose concentration threshold is employed, such as the $1.94 \mathrm{mmol} / \mathrm{L}$ recommended by the American Academy of Pediatrics for infants beyond $4 \mathrm{~h}$ of age [11], then the incidence of hypoglycaemia is markedly reduced (from 52 to $13 \%$ in our dataset). This results in a cost saving using non-enzymatic glucometers, as far fewer results require laboratory confirmation, but all patients with blood glucose concentrations between 1.94 and $2.6 \mathrm{mmol} / \mathrm{L}$ will remain untreated unless symptoms develop. Although we chose to use a lower but recommended blood glucose concentration threshold for this sensitivity analysis, these findings should be interpreted with caution, because the incidence data are taken from a population screened and treated with the aim of maintaining a blood glucose $\geq 2.6 \mathrm{mmol} / \mathrm{L}$, which is likely to have considerably reduced the number of measurements $<1.94 \mathrm{mmol} / \mathrm{L}$. In a population screened and treated entirely according to the American Academy of Pediatrics guidelines, fewer infants would have been treated and the incidence of hypoglycaemia is therefore likely to have been higher than in our sensitivity analysis, thus reducing the cost difference between enzymatic and nonenzymatic screening methods.

The use in clinical practice of an even higher blood glucose concentration threshold such as recommended by the Pediatric Endocrine Society [12] would increase the accuracy of non-enzymatic glucometers, but would require a greater number of repeat blood samples and laboratory analyses for confirmation [27], as more infants will have a blood glucose concentration that falls below the higher threshold. Overall, that practice would be likely to increase costs as well as increase the number of invasive procedures and potentially introduce delays in management.

There are a number of limitations to this study. We employed a number of simplifications in the decision tree model. These include reducing the number of potential permutations of blood glucose concentration results down to a selection of representative cases to avoid a geometric increase in decision tree branches.

We also did not subject the costs of consumables to sensitivity analysis, as they were able to be provided with relative precision, with a good level of agreement when provided from multiple sources, and with a consistent ranking and relative magnitude of difference when compared between the scenarios modelled.

Excluded from this analysis were costs related to staff training and to initial device purchase or lease, depreciation, and maintenance and calibration, on the basis that these costs are shared across an array of tests beyond glucose and/or neonatal hypoglycaemia alone, and are small compared to glucose-testing-specific consumables and associated staff time. Costs of leasing or purchasing enzymatic glucometers varies significantly depending on the device selected as well as local pricing and institutional contract relationships (for example, a second device may be heavily discounted for existing customers). Even without these potential discounts, we estimate that the difference in the initial cost of purchase between enzymatic and non-enzymatic glucometers would be offset by the savings achieved over as few as approximately 380 infants screened.

We also excluded from our model any additional costs incurred in transporting samples to the laboratory for repeat testing after screening using a non-enzymatic glucometer, although these costs may be substantial, particularly if additional staff time is involved such as use of a hospital orderly. Data were censored where an infant was admitted to NICU, at which time costs of glucose monitoring become very small compared with the overall cost of care.

Finally, the time horizon we selected for this analysis does not take into account the costs associated with medium- or long-term outcomes of neonatal hypoglycaemia. Our analysis also did not examine the extent to which screening for neonatal hypoglycaemia with a less accurate device, and the over-treatment or under-treatment that may result from that, might alter long-term outcomes. Although the costs associated with any im- 
paired neurodevelopmental outcomes are an important consideration when selecting any screening device, in the case of neonatal hypoglycaemia, in practice decisions about purchase of screening devices are commonly made on the basis of short-term costs in the absence of reliable data about long-term outcomes.

Non-enzymatic glucometers are widely used to screen for neonatal hypoglycaemia. It is hard to imagine another scenario in medicine where screening to guide diagnosis and management of a brain-threatening condition is commonly undertaken using an instrument known to be inaccurate and requiring abnormal results to be repeated, and which is justified primarily on the basis of apparently lower short-term costs. Our analysis shows that even under conservative conditions, a screening approach using enzymatic glucometers is likely to have lower direct costs, and also avoids the longer-term risks and costs associated with false-positive and false-negative results. In view of their lower cost under most circumstances and greater accuracy, enzymatic glucometers should be routinely utilised for point-of-care screening for neonatal hypoglycaemia.

\section{Acknowledgements}

We are grateful to the children and families who participated in this study. We also acknowledge the contribution of all members of (1) the CHYLD Study team: Judith Ansell, Coila Bevan, Jessica Brosnahan, Ellen Campbell, Tineke Crawford, Kelly Fredell, Karen Frost, Claire Hahnhaussen, Safayet Hossin, Greg Gamble, Anna Gsell, Yannan Jiang, Kelly Jones, Sapphire Martin, Neil Micklewood, Chris McKinlay, Grace McKnight, Christina McQuoid, Janine Paynter, Raquel O. Rodrigues, Jenny Rogers, Kate Sommers, Heather Stewart, Anna Timmings, Jess Wilson, and Rebecca Young, Liggins Institute, University of Auckland; Nicola
Anstice, Jo Arthur, Susanne Bruder, Arijit Chakraborty, Robert Jacobs, Gillian Matheson, Nabin Paudel, and Tzu-Ying (Sandy) $\mathrm{Yu}$, Department of Optometry and Vision Science, University of Auckland; Nataliia Burakevych, Department of Paediatrics, Child and Youth Health, University of Auckland; Judith Ansell and Ryan San Diego, Department of Psychological Medicine, University of Auckland; Matthew Signal and Aaron Le Compte, Department of Engineering, University of Canterbury; and Max Berry, Arun Nair, Ailsa Tuck, Alexandra Wallace, and Phil Weston, Department of Paediatrics, Waikato Hospital, Hamilton, New Zealand; (2) the CHYLD Steering Group: Jane Alsweiler, Department of Paediatrics, Child and Youth Health, University of Auckland; J. Geoffery Chase, Department of Engineering, University of Canterbury; Jane Harding, Liggins Institute, University of Auckland; Deborah Harris, Newborn Intensive Care Unit, Waikato District Health Board; Benjamin Thompson, Department of Optometry and Vision Science, University of Auckland; and Trecia Ann Wouldes, Department of Psychological Medicine, University of Auckland, Auckland, New Zealand; and (3) the International Advisory Group: Heidi Feldman, Stanford University School of Medicine, USA; William Hay, University of Colorado School of Medicine, USA; Darrell Wilson, Stanford University School of Medicine, USA; and Robert Hess, McGill Vision Research Unit, Department of Ophthalmology, McGill University, USA.

The Sugar Babies Study was funded by the Waikato Medical Research Foundation (171), the Auckland Medical Research Foundation (1110009), the Maurice and Phyllis Paykel Trust, the Health Research Council of New Zealand (09/095), and the Rebecca Roberts Scholarship. The Follow-up Study was funded by the Health Research Council of New Zealand (10/399) and the Eunice Kennedy Shriver National Institute of Child Health and Human Development of the National Institutes of Health (R01HD0692201). The content is solely the responsibility of the authors and does not necessarily represent the official views of the funding bodies.

\section{Disclosure Statement}

The authors have no conflicts of interest to disclose.

\section{References}

1 Lucas A, Morley R, Cole TJ: Adverse neurodevelopmental outcome of moderate neonatal hypoglycaemia. BMJ 1988;297:1304-1308.

2 Boluyt N, van Kempen A, Offringa M: Neurodevelopment after neonatal hypoglycemia: a systematic review and design of an optimal future study. Pediatrics 2006;117:2231-2243.

3 Harris DL, Weston PJ, Harding JE: Incidence of neonatal hypoglycemia in babies identified as at risk. J Pediatr 2012;161:787-791.
4 Hay WW Jr, Raju TN, Higgins RD, Kalhan SC, Devaskar SU: Knowledge gaps and research needs for understanding and treating neonatal hypoglycemia: workshop report from Eunice Kennedy Shriver National Institute of Child Health and Human Development. J Pediatr 2009;155:612-617.

5 Canadian Paediatric Society: Screening guidelines for newborns at risk for low blood glucose. Paediatr Child Health 2004;9:723-740.

6 Kerstjens JM, Bocca-Tjeertes IF, de Winter AF, Reijneveld SA, Bos AF: Neonatal morbidities and developmental delay in moderately preterm-born children. Pediatrics 2012;130: e265-e272.
7 Burns CM, Rutherford MA, Boardman JP, Cowan FM: Patterns of cerebral injury and neurodevelopmental outcomes after symptomatic neonatal hypoglycemia. Pediatrics 2008; $122: 65-74$

$\checkmark 8$ Pildes RS, Cornblath M, Warren I, Page-El E, Di Menza S, Merritt DM, Peeva A: A prospective controlled study of neonatal hypoglycemia. Pediatrics 1974;54:5-14.

9 Montassir H, Maegaki Y, Ogura K, Kurozawa Y, Nagata I, Kanzaki S, Ohno K: Associated factors in neonatal hypoglycemic brain injury. Brain Dev 2009;31:649-656. 
10 Caksen H, Guven AS, Yilmaz C, Unal O, Basaranoglu M, Sal E, Kaya A: Clinical outcome and magnetic resonance imaging findings in infants with hypoglycemia. J Child Neurol 2011;26:25-30.

11 Committee on Fetus and Newborn, Adamkin DH: Postnatal glucose homeostasis in latepreterm and term infants. Pediatrics 2011, 127:575-579.

12 Thornton PS, Stanley CA, De Leon DD, Harris D, Haymond MW, Hussain K, Levitsky LL, Murad MH, Rozance PJ, Simmons RA, Sperling MA, Weinstein DA, White NH, Wolfsdorf JI; Pediatric Endocrine Society: Recommendations from the Pediatric Endocrine Society for Evaluation and Management of Persistent Hypoglycemia in Neonates, Infants, and Children. J Pediatr 2015;167:238245.

$\checkmark 13$ Cornblath M, Hawdon JM, Williams AF, Aynsley-Green A, Ward-Platt MP, Schwartz R, Kalhan SC: Controversies regarding definition of neonatal hypoglycemia: suggested operational thresholds. Pediatrics 2000;105: 1141-1145.

14 Harris DL, Weston PJ, Battin MR, Harding JE: A survey of the management of neonatal hypoglycaemia within the Australian and New Zealand Neonatal Network. J Paediatr Child Health 2014;50:E55-E62.
15 Lucas A: Outcome of neonatal hypoglycaemia. BMJ 1999;318:194.

16 Ho HT, Yeung WK, Young BW: Evaluation of "point of care" devices in the measurement of low blood glucose in neonatal practice. Arch Dis Child Fetal Neonatal Ed 2004; 89:F356-F359.

17 Dixon KC, Ferris RL, Marikar D, Chong M, Mittal A, Manikam L, Rose PJ: Definition and monitoring of neonatal hypoglycaemia: a nationwide survey of NHS England Neonatal Units. Arch Dis Child- Fetal Neonatal Ed 2017;102:F92-F93.

18 Bellini C, Serra G, Risso D, Mazzella M, Bonioli E: Reliability assessment of glucose measurement by HemoCue analyser in a neonatal intensive care unit. Clin Chem Lab Med 2007; 45:1549-1554.

19 Edlin R, McCabe C, Hulme C, Hall P, Wright J: Cost Effectiveness Modelling for Health Technology Assessment: A Practical Course. Heidelberg, Adis, 2015.

20 Harris DL, Weston PJ, Signal M, Chase JG, Harding JE: Dextrose gel for neonatal hypoglycaemia (the Sugar Babies Study): a randomised, double-blind, placebo-controlled trial. Lancet 2013;382:2077-2083.
21 Auckland District Health Board: Guidelines for the Management of Hypoglycaemia. http://www.adhb.govt.nz/newborn/Guidelines/Nutrition/HypoglycaemiaManagement.htm (accessed June 28, 2016).

22 New Zealand Nurses Organisation: Collective Agreements 2016. http://www.nzno.org.nz/ support/collective_agreements (accessed October 1, 2016).

23 Ranger M, Chau CM, Garg A, Woodward TS, Beg MF, Bjornson B, Poskitt K, Fitzpatrick K, Synnes AR, Miller SP, Grunau RE: Neonatal pain-related stress predicts cortical thickness at age 7 years in children born very preterm. PLoS One 2013;8:e76702.

-24 Blomquist HK, Jonsbo F, Serenius F, Persson LA: Supplementary feeding in the maternity ward shortens the duration of breast feeding. Acta Paediatr 1994;83:1122-1126.

25 Park S, Kim BN, Kim JW, Shin MS, Yoo HJ, Cho SC: Protective effect of breastfeeding with regard to children's behavioral and cognitive problems. Nutr J 2014;13:111.

26 Bar S, Milanaik R, Adesman A: Long-term neurodevelopmental benefits of breastfeeding. Curr Opin Pediatr 2016;28:559-566.

27 Beardsall K: Measurement of glucose levels in the newborn. Early Hum Dev 2010;86:263267. 